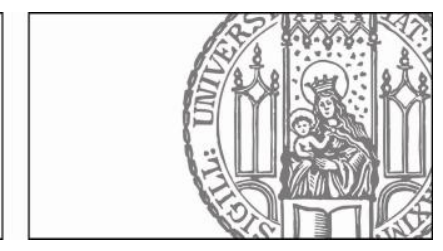

\title{
Preventing Dropout in Research, Assessment and Treatment with Refugees - a guideline (V01 - 15.09.2020)
}

Project "Predicting and Preventing Dropout in Research, Assessment and Treatment with Refugees (PrevDrop)" (BMBF-funded, FKZ: 01EF1901)

\section{Verena Semmlinger, Thomas Ehring}

Lehrstuhl für Klinische Psychologie und Psychotherapie

Department Psychologie

Ludwig-Maximilians-Universität München

Leopoldstraße 13

80802 München 


\section{Table of Content}

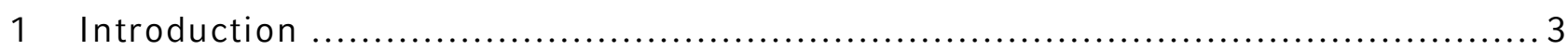

1.1 Definition of Dropout .......................................................... 5

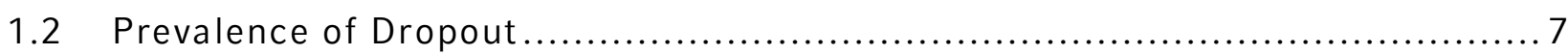

1.3 Relevance of considering dropout in research and treatment................... 8

2 Predictors and subjective reasons for dropout ................................... 9

2.1 General research findings on predictors for dropout.......................... 9

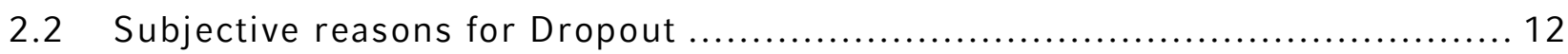

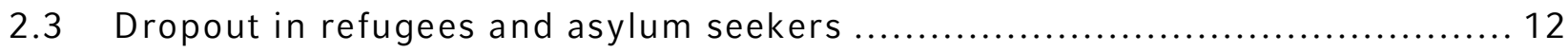

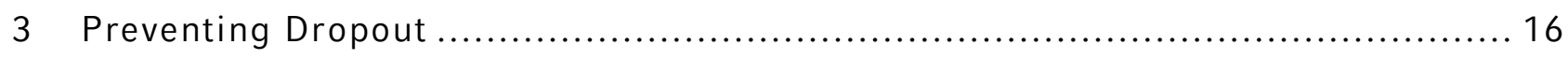

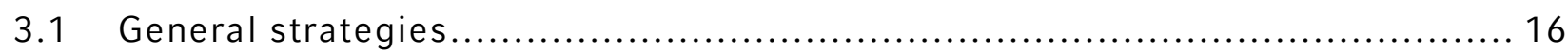

3.2 Specific strategies for refugees and asylum seekers ........................... 19

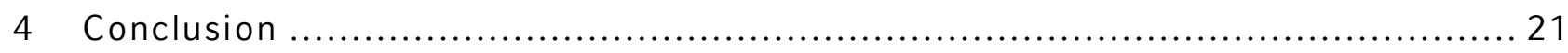

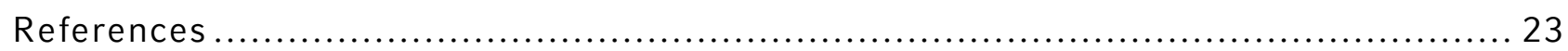

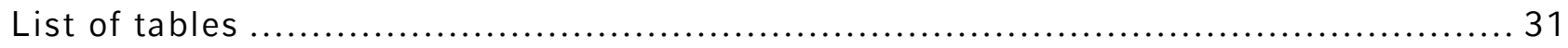




\section{Introduction}

"We live in an increasingly diverse world in which migration is both a current issue and one for the years to come. The growth in migrant numbers arriving in Europe creates challenges that require a rapid humanitarian response and put pressure on health systems."

Zsuzsanna Jakab, WHO Regional Director for Europe (Priebe et al., 2016)

Millions of people are forced to flee their home country due to war, persecution, conflicts, or human rights violations. According to the latest report of the United Nation High Commissioner for Refugees (UNHCR), there are 70.8 million forcibly displaced people worldwide (UNHCR, 2019). With an increase of 2.3 million people compared to the previous year, the global number of forcibly displaced people by the end of 2018 is the highest the UNHCR has registered in their 70-year existence.

The term forcibly displaced people includes refugees (20.4 million under UNHCR's mandate, plus 5.5 million Palestinian refugees under the United Nations Relief and Works Agency's (UNRWA) mandate), asylum seekers (3.5 million), and internally displaced people (41.3 million) who were forced to flee but did not leave their countries (UNHCR, 2019). Therefore, when referring to forcibly displaced people a distinction between these different groups is needed. The majority of the worldwide refugees (67\%) come from Syria, Afghanistan, South Sudan, Myanmar, and Somalia. With the fifth largest refugee population worldwide, Germany plays an important part as a host country (UNHCR, 2019).

The trends reported by the UNHCR indicate that the refugee crisis is a global challenge that demands the attention of the European Union. A refugee population of 6.5 million (1.06 million in Germany; UNHCR mandate) and a total number of pending asylum cases of 1.3 million has been reported for Europe, with 161,900 new asylum applications in Germany (third largest recipient worldwide) followed by France (114,500 new claims) by the end of 2018 (UNHCR, 2019).

Refugees and asylum seekers face a number of stressful events and threatening experiences. These include experiences prior to migration such as persecution, multiple traumatic experiences caused by armed conflicts, war, torture, or economic hardship (Bogic et al., 2012; Böttche et al., 2016; Hargreaves, 2002; Hollifield et al., 2002; Kalt et al., 2013; Priebe et al., 2016) as well as experiences during the displacement process, such as physical harm, life-threatening conditions, and separation from family members (Böttche et al., 2016; Priebe et al., 2016; Ryan et al., 2008). Furthermore, resettling in a new country can provoke various post-migration stressors. These include challenging accommodation situations, poor socio-economic conditions, uncertainty, fear of detention, experiences of discrimination, language difficulties, social isolation, or complications in the asylum-application process (Böttche et al., 2016; Liedl et al., 2016; Porter \& Haslam, 2005; Priebe et al., 2016). 
The exposure to pre-, peri- and post-migration stressors causes a burden that might affect the physical as well as the mental health of refugees and asylum seekers. There is strong research evidence indicating considerable rates of psychological disorders among this population (Nickerson et al., 2011). In their comprehensive meta-analysis with a large sample of refugees from various conflict-affected areas and in a range of resettlement countries, Steel et al. (2009) report a prevalence rate of 30.6\% [26.3\% - 35.2\%] for posttraumatic stress disorder (PTSD) and $30.8 \%[26.3 \%-35.6 \%]$ for depression. For both, PTSD $(0 \%-99 \%)$ and depression $(3 \%-$ $85.5 \%$ ), the variance of prevalence rates among studies was very high. Focusing on refugees resettled in Western countries, the prevalence of PTSD and depression reported by Fazel et al. (2005) is $9 \%[8 \%-10 \%]$ and $5 \%[4 \%-6 \%]$, respectively. It is worth noting that the majority of studies considered refugees from southeast Asia or former Yugoslavia. More recent studies paint an even more precarious picture. Among Syrian refugees, for example, a prevalence rate of 33.5\% for PTSD (Alpak et al., 2015) and 29.5\% for depression (Gammouh et al., 2015) has been estimated. A systematic review focusing on Iraqi refugees living in Western countries yielded prevalence rates ranging from 8 to $37.2 \%$ for PTSD and 28.3 to $75 \%$ for depression (SlewaYounan et al., 2015). Turrini et al. (2017) provide an overview of systematic reviews reporting prevalence rates for PTSD, depression, and anxiety disorder in refugees and asylum seekers resettled in low -, middle- and high-income countries. For PTSD prevalence rates ranged from 3 $50 \%$ when considering a resettlement in high-income countries (1), from $0.2-76.5 \%$ when considering low- and middle income countries (2) and $0-99 \%$ when considering all three (3). For depression, the rates were the following $3-100 \%$ (1), $2-89.5 \%$ (2), $2.3-100 \%$ (3). For anxiety: $12-77 \%$ (1), $4-81.6 \%, 5-90 \%$ (3). Even though the substantial variability in the reported prevalence rate is important to be considered, overall finding indicate, that prevalence rates for depression and anxiety were as high as for PTSD. The need to take the mental health of refugees and asylum seekers into consideration is highlighted through this umbrella review of systematic reviews (Turrini et al., 2017).

The presence of mental health burdens among refugees and asylum seekers results in a substantial demand of adapted and effective mental health interventions and treatment services for this group. An expanding body of research has examined the effectiveness of various psychological or psychosocial interventions for refugees and asylum seekers over the past decades, with the majority of studies focusing on PTSD symptoms. Several reviews on this topic indicate that psychological and psychosocial interventions were effective in reducing PTSD symptoms as well as depression (Crumlish \& O'Rourke, 2010; Kip et al., 2020; J. E. Lambert \& Alhassoon, 2015; Nose et al., 2017; Palic \& Elklit, 2011; Thompson et al., 2018; Turrini et al., 2019). Further reviews taking a wider range of mental diseases into account suggest the effectiveness of psychosocial interventions in decreasing PTSD, depression, and anxiety symptoms and further reveal the persistency of these positive effects at follow-ups (Tribe et al., 2019; Turrini et al., 2019). 
When evaluating treatments for refugees, it appears important not only to evaluate the efficacy of treatment effects on symptom reduction. Instead, the acceptability, feasibility and availability of treatments are important additional outcomes. Can refugees with mental health problems gain access to treatment? Do their symptoms improve? But also, do individuals remain in a planned treatment? How frequently do refugees drop out of treatment?

Despite the growing body of literature on the effectiveness of psychological interventions for refugees and asylum seekers, the empirical research on dropout is limited. In studies reporting dropout rates in refugees receiving psychosocial treatment, this variable has most frequently been assessed as a secondary outcome only and/or investigated in additional post-hoc analyses of data sets that were mainly focused on evaluating treatment efficacy. To our knowledge, there is currently no published systematic review or meta-analysis assessing the prevalence, predictors, or established preventive measures for dropout in psychological or psychosocial interventions for refugees and asylum seekers.

\subsection{Definition of Dropout}

There is currently no consensus in the literature on the definition of dropout. Many authors define dropout as the termination of an initiated treatment before the problems (symptoms, impairment of the functional level, suffering) that had led to the start of the intervention have been alleviated (Garfield, 1986; Hatchett \& Park, 2003; Swift et al., 2009; Swift \& Greenberg, 2012). Swift and Greenberg (2012) specify this broad definition. According to the authors, it implies a termination of the treatment without fulfilment of the therapeutic goals, without attainment of the full therapeutic benefit that would have been possible with normal termination of the therapy or without completion of the full scope of the therapy (see Table 1). It is important that the decision on the termination of the treatment is made exclusively by the patient and without mutual consent (Horner \& Diamond, 1996; Swift \& Greenberg, 2012).

Other authors just concentrate on parts of this specific definition made by Swift and Greenberg (2012). Thus some speak of dropout when a planned therapy duration, i.e. a fixed number of therapy sessions or a fixed duration in months, cannot be achieved (e.g. Beckham, 1992; Gunderson et al., 1989). Others focus on fulfilling the treatment protocol (e.g. Maher et al., 2010), or define dropout as a non-appearance at two consecutive scheduled sessions or the last scheduled session (e.g. Hatchett et al., 2002; Kolb et al., 1985). Importantly, in all definitions, dropout is distinguished from regular termination of treatment and the refusal of therapy (Garfield, 1994).

To operationalize dropout, studies use different methods based on the definition used by the respective authors (see Table 1 for an overview). First, a specific number of therapy sessions can be defined, which is considered the minimum therapy dose for symptom improvement. Failure to meet this minimum number is then considered a dropout (M. J. Lambert, 2007; Swift \& Greenberg, 2012). Second, a different operationalization considers failure to comply with the 
treatment protocol to be decisive, i.e. anyone who does not comply with the entire protocol is counted as a dropout (Swift \& Greenberg, 2012). Third, dropout is sometimes operationally defined as any instance where a patient misses a scheduled therapy session without rescheduling it or coming to further sessions (Swift \& Greenberg, 2012). Forth, there is an operationalization method purely focusing on the therapist's judgement, i.e., after treatment termination, the therapist decides whether the therapy is considered prematurely terminated (Swift \& Greenberg, 2012). Fifth, decision making on dropout can be based on clinical significance. Dropout is any discontinuation of therapy without demonstrable improvement and without achieving a score in the normal range during the outcome measurement (Hatchett \& Park, 2003).

The choice of a particular method of operationalizing dropout depends on the setting and the goal of the study or treatment and each has a different set of advantages and disadvantages. While, for example, in the natural therapy setting, duration-based operationalization methods such as a minimal therapy dose (point 1) or the completion of a treatment protocol (point 2) may seem problematic in some cases, it is advisable in RCTs with a predetermined number of treatment sessions (Zimmermann, 2016).

Wierzbicki and Pekarik (1993) advocate the therapist's judgment as the preferred operationalization method for dropout. In contrast, Swift and Greenberg (2012) abstain from favoring one specific method; instead they recommend a combination of two types of operationalization, for example combining the therapist's judgement with a more objective technique. However, they emphasize the importance for the field to start using a uniform definition and operationalization method for dropout (Swift \& Greenberg, 2012).

It should be noted that the variability in the definition and operationalization of dropout across studies makes it difficult to compare and interpret findings.

Based on the current literature, it seems reasonable to make different recommendations depending on context and objectives for assessing dropout. In clinical practice, we recommend the therapist's judgement as the main method that is to be supplemented by a more objective procedure. Due to the importance of regular monitoring of the treatment progress, a dropout decision based on clinically significant change appears to be an adequate complementary method. In clinical research, we recommend a more objective method that it feasible and allows comparability. In our view, a duration/ dose-based operationalization method should be favored, i.e., defining a specified number of sessions that need to be attended to be classified as a completer. 
Table 1: Definition and operationalization of dropout

\begin{tabular}{|c|c|}
\hline \multicolumn{2}{|c|}{ Definition of dropout and its implications } \\
\hline \multicolumn{2}{|c|}{ Dropout is termination of treatment without, ... } \\
\hline & fulfillment of a therapeutic goal \\
\hline & attainment of full therapeutic benefit \\
\hline & completion of the full scope of therapy \\
\hline Definition & Operationalization methods \\
\hline $\begin{array}{l}\text { Duration-based/ } \\
\text { minimum dose }\end{array}$ & Fixed number of therapy sessions \\
\hline Treatment protocol & Failure to comply the entire treatment protocol \\
\hline $\begin{array}{l}\text { Missed } \\
\text { appointments }\end{array}$ & $\begin{array}{l}\text { Miss of a scheduled therapy session without rescheduling or coming to } \\
\text { further sessions }\end{array}$ \\
\hline $\begin{array}{l}\text { Therapist } \\
\text { judgement }\end{array}$ & Dropout decision based on the therapist's judgement \\
\hline Clinical significance & $\begin{array}{l}\text { Termination of therapy without clinically significant improvement and } \\
\text { without achieving a score in the normal range during outcome } \\
\text { measure }\end{array}$ \\
\hline
\end{tabular}

\subsection{Prevalence of Dropout}

A number of meta-analyses have been published reporting on the prevalence of dropout from psychological treatment.

Wierzbicki and Pekarik (1993) conducted the first comprehensive, systematic statistical review of dropout rates in psychological treatment. Across 125 studies they determined an average dropout rate of $46.86 \%(95 \% \mathrm{Cl}=[42.9 \%-50.82 \%])$, which is in line with earlier reviews consistently reporting a dropout rate in the range of 50\% (Baekeland \& Lundwall, 1975; Garfield, 1986; Wierzbicki \& Pekarik, 1993). More recent meta-analyses, on the other hand, paint a slightly different picture. In their large-scale meta-analysis on dropout from psychological treatment with 669 included studies and a total of 83,834 examined adult patients, Swift and Greenberg (2012) reported a weighted mean dropout rate of $19.7 \%(95 \% \mathrm{Cl}[18.7 \%-20.7 \%])$. However, there was a high degree of heterogeneity among the included studies, with dropout rates ranging from $0 \%$ to $74.23 \%$ (Swift \& Greenberg, 2012). Other recent comprehensive reviews are in line with these findings. For example, in a meta-analysis conducted by Fernandez et al. (2015) the average dropout rate was $26.2 \%$ and in a recent review aiming at a comparison between psychological treatment and pharmacotherapy Swift et al. (2017) found an overall treatment dropout rate of $21.9 \%$. 
The dropout rate appears to be moderated by the diagnosis of the client. Studies with no specification of the treatment-focus on one diagnosis, or with a treatment-focus on a nonclassified category reported the highest dropout rates (27.7\%) (Swift \& Greenberg, 2012). Second and third largest dropout rates can be found for personality disorder treatments $(25.6 \%)$ and eating disorder therapy (23.9\%). Further meta-analysis, concentrating on the treatment dropout in specific groups of patients report, for example, an average dropout rate of $37 \%$ in personality disorder treatments (McMurran et al., 2010), or a dropout rate of $18.28 \%$ in treatments for PTSD in general and 36\% for trauma-specific treatments (Imel et al., 2013).

Some analyses have also examined whether dropout rates are related to specific treatment approaches. There is some evidence showing significant differences in dropout between treatment approaches only in the context of depression, eating disorders and PTSD (Swift $\&$ Greenberg, 2014). For depression and PTSD, the lowest dropout rate was reported in integrative approaches that combined several techniques without referring to one broad orientation such as cognitive-behavioral or psychodynamic (10.9\% and $8.8 \%)$. Dialectical-behavior therapy (DBT) resulted in the lowest dropout rate for eating disorders (5.9\%). For the remaining nine diagnostic categories examined, no significant difference in dropout rates for the separate approaches could be detected, indicating that general therapeutic treatment factors (e.g. therapeutic alliance), rather than specific methods might be decisive for retention in treatment (Swift \& Greenberg, 2012, 2014).

\subsection{Relevance of considering dropout in research and treatment}

Premature termination of psychological treatment can cause various adverse consequences for the patients, the therapist as well as the public healthcare system. Considering the negative consequences for patients dropping out of treatment, there is a reduced probability of improvement in the psychosocial functional level and in the symptoms that led them to seek treatment (Bjork et al., 2009; Cahill et al., 2003; Swift et al., 2012). Furthermore, most patients who have decided to drop out of a therapy state that they have been very dissatisfied with the therapy (Bjork et al., 2009). In addition, premature termination of treatment can also affect the therapist. Several authors highlight the long-term complex and persistent effects of therapy discontinuation on the therapist. As a fundamental stressor for the therapist (Farber, 1983), premature discontinuation of therapy can lead to a reduction in self-esteem and to feelings of frustration, failure, and rejection (Ogrodniczuk et al., 2005; Piselli et al., 2011). Additionally, the burden of multiple therapy dropouts on the mental health system must not be forgotten. The premature discontinuation of therapy affects the health care system on several levels (Barrett et al., 2008). These include the additional strain on already scarce resources and the ongoing costs to the health care system caused by a prolonged illness (Barrett et al., 2008; Parsonage, 2003; Swift et al., 2012). 
In view of the negative consequences of premature discontinuation of therapy, it is of great importance to take a comprehensive look at the phenomenon of dropout. In additional to establishing the prevalence of patients' dropping out from treatment, it appears of particular interest to identify predictors and risk factors for dropout, and develop strategies for the reduction of dropout in research and practice. Although systematic reviews and meta-analyses on these phenomena are emerging see e.g. Swift \& Greenberg (2012) for an influential large-scale review and meta-analysis as well as Cinkaya (2016) for a meta-analysis in the German-speaking context.

Reviews specially focusing on dropout in research, assessment and treatment of refugees and asylum seekers are missing. The current guideline therefore focuses on aims to inform researchers and clinicians working with refugees about the current state of evidence regarding the prevalence, prediction, and reduction of dropout. In each of the following chapters, we will first summarize evidence regarding dropout from psychological treatment in general, while then specifically focusing on refugee populations in a second step.

\section{Predictors and subjective reasons for dropout}

For a more comprehensive understanding of the phenomenon and in order to develop strategies that can reduce its occurrence, it is essential to understand the variables that lead to premature discontinuation of psychological treatment. Further, a differentiation between predictors and subjective reasons for terminating a treatment prematurely is required (Swift et al., 2012).

In the following, an overview of empirical evidence regarding predictors and reasons for dropout will be provided. While the individual aspects are initially described in detail, they are summarized in tabular form at the end of this chapter (see Table 2).

\subsection{General research findings on predictors for dropout}

A number of predictors of dropout can be identified. These predictors do not present a uniform picture, but rather vary across studies or therapy settings. Furthermore, there is evidence for complex interactions between the variables.

\section{Patient variables}

\section{Sociodemographic Variables}

In the currently largest meta-analysis on this topic, Swift and Greenberg (2012) found client's age as the only consistent predictor of dropout across all analyses, with younger patients being more likely to prematurely terminate psychological treatment. No significant differences in dropout rates could be found for patients' ethnicity or employment status. Due to the different findings depending on the type of statistical analysis, no clear statements can be made about the influence of educational level, gender, and marital status. While a comparison of dropouts and completers 
showed a significant difference in education (dropouts being less educated), but not in gender or marital status, meta-regression analyses contradict these results by revealing significant differences for gender (higher dropout, smaller percentage of females in sample) and marital status (higher dropout, fewer patients in committed relationships), but not education (Swift $\varepsilon$ Greenberg, 2012).

While some reviews are in line with the findings on the impact of patient's age on the dropout rate (Barrett et al., 2008; Winkler, 2018), Zimmermann et al. (2017) could not confirm this association. However, they reported a significant impact of gender (more dropout in male patients) and level of education (higher dropout in less educated) (see Table 2 for an overview).

\section{Psychological Variables}

Considering the influence of the patient's diagnosis on premature termination, Swift and Greenberg (2012) report dropout rates to be moderated by diagnoses, with the highest dropout rate in the treatment of patients with personality disorders or eating disorders. McMurran et al. (2010) confirm these findings by pointing out the high dropout rate in treatments of personality disorders. Studies with German samples are in line with these findings. For example, in their analysis of dropout in an outpatient therapy setting Cinkaya (2016) report that patients with borderline personality disorder as well as other personality disorders where more likely to discontinue therapy prematurely. In contrast, the lowest dropout rate was found among patients with depressive, somatoform, and anxiety disorders (Cinkaya, 2016). When considering personality style, a significant association with compulsive and histrionic personality dimensions can be shown in that a high degree of compulsive accentuations of personality is associated with smaller dropout rates, whereas histrionic patients tend to dropout more frequently (Zimmermann et al., 2017).

While most studies have focused on sociodemographic variables and diagnoses, some additional moderators have been suggested. In their comprehensive literature review, Barrett et al. (2008) point out the influence of the severity and complexity of diagnoses, that is for example Axis II comorbidity, on dropout rates. Further, the level of psychological mindedness (negative correlation with dropout), as well as positive perception of mental health and mental health treatment (negative correlation with dropout), as well as current treatment expectation are associated with the dropout rates (Barrett et al., 2008). In addition, Zimmermann et al. (2017) found that high dropout was associated with a higher initial impairment and a lower therapy expectation, i.e. lower confidence that treatment will help in solving their problems. 


\section{Treatment variables}

A number of variables related to the treatment provided are potentially predictive for dropout (see Table 2). Swift and Greenberg (2012) reported significantly lower dropout rates in treatments with fixed number of sessions (time-limited) and high levels of manualization. There were also differences in treatment settings, with a higher dropout rate in university-based institutions. Interestingly, no significant difference in terms of treatment orientation or treatment format (group, individual) could be identified. However, differences in dropout between different type of treatments may be moderated by disorder, with evidence for larger effects of treatment type on dropout in depression, eating disorder, and PTSD (Swift \& Greenberg, 2014).

According to Barett et al. (2008), treatment barriers have a significant influence on the probability of terminating therapy prematurely. The authors refer to earlier studies hypothesizing difficulties in finding a therapy, a long waiting list, as well as long commutes to therapy locations as possible predictors of dropout.

\section{Therapist-/ provider variables}

Level of therapist experience has been shown to be a predictor of dropout (Swift \& Greenberg, 2012). Among patients treated by therapists in training, dropout rates are significantly higher than when treated by an experienced therapist. No significant difference in dropout rates have been found for therapists' age, gender, or race. Roos and Werbart (2013) confirmed the connection between higher experience levels and dropout rates in their review. In this context, the impact of the quality of the therapeutic alliance should be emphasized. A large number of studies can be identified that have found a connection between strength of the therapeutic alliance and dropout probability, with weaker alliances tending to predict dropout (Cinkaya, 2016; Roos \& Werbart, 2013; Sharf et al., 2010). Further, Zimmermann et al. (2017) underline the importance of the so-called therapist effect when examining dropout. In their study, they reported that $5.72 \%$ of the variance in dropout could be explained through therapist differences (therapist effect).

\section{Study and design variables}

In addition to variables related to characteristics of patients, therapists, or the therapy itself, it is further interesting to consider the study design, i.e. how the dropout rate is influenced by methodological factors, such as type and conduct of studies (see Table 2).

Swift and Greenberg (2012) demonstrated that dropout rates were significantly moderated by the definition of dropout used in the studies, with the highest dropout rates for therapist judgment. These results are in line with the findings reported by Wierzbicki and Pekarik (1993) or Hatchett and Park (2003). Furthermore, study type has a significant influence on determined rates of dropout (Swift \& Greenberg, 2012). The dropout rates reported by studies of treatment 
effectiveness were significantly higher than those, reported from studies focusing on efficacy. In contrast, there was no significant effect of the publication year of studies.

\subsection{Subjective reasons for Dropout}

While a growing body of research is examining predictors for dropout, comparatively little is known about subjective reasons reported by patients for their dropping out of treatment (for an overview, see Table 2). The first source of information on subjective reasons for dropout are surveys asking therapists or patients to rate possible reasons for dropout specified a priori. Insufficient motivation for therapy and/ or for change proved to be the most frequent reason for discontinuation documented by therapists (Cinkaya, 2016). Therapists also reported that patients often suddenly stop attending therapy session and no further contact is possible, therefore reasons for discontinuation often remain unknown. Patients, on the other hand, frequently report dissatisfaction with the therapist and the therapy as well as external barriers as reasons for dropout in these surveys (Westmacott et al., 2010).

Additionally, a number of studies have used qualitative designs to generate hypotheses on possible reasons for termination. In their qualitative analysis of patient interviews on premature termination, Knox et al. (2011) reported unresolvable ruptures between patients and therapists as frequently cited reasons for dropout from a patients' perspective. Furthermore, patients indicated to have stopped the therapy for financial and logistic reasons. The end of therapy itself was mostly sudden and carried out unilaterally by the patient. In an older qualitative study patients reported that a lack of improvement of their problems, dissatisfaction with the therapy, and external obstacles had mainly led to therapy discontinuation (Pekarik, 1992). Consistent with these findings, Westmacott et al. (2010) describe external barriers and dissatisfaction as the primary reasons for discontinuing therapy, but also emphasize that some patients terminate therapy prematurely because they are already satisfied with the therapeutic progress.

According to Swift and Greenberg (2012), these different reasons for premature termination of therapy can be understood as the result of a cost-benefit calculation. In this calculation, the patient compares the perceived and anticipated costs of continuing the therapy with the perceived and anticipated benefits of the therapy. If the costs exceed the anticipated benefit, dropout becomes more likely.

\subsection{Dropout in refugees and asylum seekers}

Although empirical evidence on predictors of and reasons for dropout in refugees in asylum seekers is sparse, it is conceivable that many variables identified in Western populations also apply in this specific population. However, it appears likely that additional factors that are specific for this group need to be considered (see Table 2). 
Importantly, according to numerous studies there is evidence for cultural differences in the perception of, thoughts about, and beliefs related to mental illness and psychotherapeutic treatments (Barrett et al., 2008). While physical disorders are usually explained using the Western aetiological model, Liedl et al. (2016) suggest that refugees tend to use traditional, culture-specific assumptions when explaining mental disorders. The cause of the illness is then seen, for example, in external circumstances like "the evil eye", "the curse of fellow human beings", or the "intervention of the ancestors" (Liedl et al., 2016). Furthermore several authors assume that in many cultures there is no body-soul dualism, both mental and physical pain is experienced holistically (Ebner, 2001; Liedl et al., 2016). In addition a number of studies have suggested that the socialization of individuals can influence the perception of their own mental state and the associated need for therapeutic support (Barrett et al., 2008). If one looks at the African cultural area, for example, there is a socialization-related tendency among African women to have to act as strong caregivers (Boyd-Franklin, 2013). This includes the feeling of being solely responsible for the needs of the family and thus reduces the probability of accepting external help. At the same time, men tend to be educated to suppress or hide their feelings (BoydFranklin, 2013). Unfortunately, there is insufficient research on the influence of the perception of mental illnesses on the probability of dropout. If at all, this factor is only investigated in the context of stigmatization, yet again the results are inconsistent (Barrett et al., 2008).

It should be noted that not only the perception of mental health, but also the perception and knowledge of psychotherapeutic treatment and therapists could have an influence on starting, and also on prematurely terminating therapy. This attitude towards psychological treatment appears to be influenced by socialization and cultural differences as well (Barrett et al., 2008; Priebe et al., 2016). In their qualitative study with 48 interviewed mental health care professionals in 16 European countries, Sandhu et al. (2013) found that many therapists stated that patients from other cultures have difficulties in building trust. This was due to both, previous negative experiences with authorities and the unfamiliarity with the health care system. These results were supported by Priebe et al. (2016) who reviewed the results of several studies giving evidence for a lack in gaining trust towards health care professionals among refuges and asylum seekers. Furthermore, there is first evidence that refugees and asylum seekers are more likely to fear exclusion or marginalization due to mental illness and psychotherapeutic treatment (Sandhu et al., 2013). Another important point stated by various authors is that the different expectations of treatment held by patients from other cultural backgrounds, can influence the occurrence of dropout from psychological treatment (e.g. Priebe et al., 2016; Sandhu et al., 2013; Slobodin \& de Jong, 2015; van Loon et al., 2011). Liedl et al. (2016) suggest for example, that traumatized refugees expect a medically or spiritually oriented therapy method that also leads to an improvement of their living conditions they consider the cause of their symptoms. Furthermore, "talking as a method of healing" is a mostly unknown concept, so the authors. In order to avoid disappointment, a comprehensive explanation of the therapy method as well as the clarification of the individual therapy expectation is therefore indispensable (Liedl et al., 2016). 
In addition to perceptions about mental health and psychotherapeutic treatments that vary across cultures, there are other important factors that may influence the risk of dropout in this people group. Refugees and asylum seekers are situated in demanding living conditions and face specific challenges, which can be seen as additional obstacles that make it difficult to remain in therapy.

Slobodin and de Jong (2015) hypothesize that language barriers and communication difficulties as well as frequently changing residences and contact details may increase the probability of dropout. Furthermore, refugees and asylum seekers often have limited economic resources. Some mental health care professionals assume that this could also influence the acceptance of psychotherapeutic treatment offers (Sandhu et al., 2013). Considering the difficulties these individuals or families face every day, it is clear that it may be difficult to attend psychological treatment regularly, if daily care for children is not guaranteed, or if one may not miss a day at work without the risk of dismissal, just to give a few examples. Many refugees and asylum seekers further lost their social environment due to resettling in another country. Several authors argue that the lack of support through social contacts, the feelings of loneliness, isolation and homesickness can lead to a sense of helplessness and fear of marginalization that may enhance the possibility of dropout (Liedl et al., 2016; Sandhu et al., 2013). Furthermore, a rejected asylum application can also inevitably lead to premature termination of therapy (Slobodin \& de Jong, 2015). Often, these specific burdens can be summarized under the terms of post-migration stressors explained above. In several reviews the authors have assumed that while the presence of post-migration stressors on the one hand increases the psychological burden and thus the need for therapeutic support, they can also be seen as obstacles to remaining in therapy (Böttche et al., 2016; Porter \& Haslam, 2005; Priebe et al., 2016). Abdallah-Steinkopff (2020) hypothesizes that the reason for this may be the feeling that psychological treatment does not help sufficiently in meeting their daily demands (asylum procedures, fear for family, poor living conditions). The author states that there is a higher perceived value in offers of support that help with current daily burdens than in therapies dealing with one's own psychological issues, such as past trauma (Abdallah-Steinkopff, 2020). 
Table 2: Key findings on predictors and subjective reasons for dropout

Predictors of dropout fall into different categories: patient; treatment; therapist; study and design. Further, consider subjective reasons.

\section{Patient}

- Sociodemographic variables:

- Younger patients are more likely to dropout (Swift, 2012) (not confirmed by Zimmermann, 2016)

- Higher dropout in less educated patients (Zimmermann, 2016) (unclear in Swift, 2012)

- Higher dropout in male patients (Zimmermann, 2016) (unclear in Swift, 2012)

- No association with: race; employment status

- Consistent findings for higher dropout rates in patients with personality disorders

- Higher dropout rates: histrionic personality style, lower dropout rates: compulsive personality style

- Further: treatment expectation; initial impairment; psychological mindedness; perception of mental health

\section{Treatment}

- Lower dropout rate: Fixed number of treatment session; high level of manualization

- Higher dropout rate: university-based institutions

- No differences: treatment format; treatment orientation (note: difference when clustering between disorder categories)

\section{Therapist}

- Higher dropout rate: therapist in training

- No differences: therapists' age, gender, or race

- Influence of strength of therapeutic alliance on dropout!

Study and design

- Significant influence of dropout definition on reported rates (highest: therapist judgment)

- Higher dropout rates: effectiveness studies

\section{Subjective Reasons}

- Insufficient motivation for therapy; insufficient motivation of change; insufficient improvement of problems/ symptoms

- Dissatisfaction with therapist or therapy; unresolvable ruptures in patient-therapist relationship

- External barriers (e.g. financial, logistical)

\section{Specific factors for refugees and asylum seekers}

- Cultural differences in perception/ thoughts/ beliefs about mental health (culture specific assumptions; socialization)

- Cultural differences in perception of and knowledge about psychotherapeutic treatment and therapists (trust; unfamiliarity with health care system)

- Differences in treatment expectations

- Specific barriers: language; communication; accommodation situation; limited economic resources; lack of social contacts; rejected asylum applications

- Presence of multiple post-migration stressors can be another factor that might enhance the possibility of dropout - because of the higher value in solving the daily burden. 


\section{Preventing Dropout}

The reduction of dropout is a major challenge in both clinical practice and clinical research. Therefore, effective measures are needed to prevent dropout in clinical practice in order to provide the best possible care for all patients. In clinical research, such implementations can increase the quality and validity of research results and thus contribute to the improvement of therapeutic measures in the long term. This appears particularly important for refugees and asylum seekers who represent a highly vulnerable group where high dropout rates are to be expected. For an overview of measures to prevent dropout, see Table 3.

\subsection{General strategies}

Based on the results of their meta-analysis on dropout and its predictors (Swift $\&$ Greenberg, 2012), Swift et al. (2012) derive various strategies to reduce dropout in therapy, regardless of the specific type of treatment. These strategies primarily refer to actions that can be applied during the first therapy sessions.

First Swift et al. (2012) suggest that therapists should inform their patients about the duration of therapy and typical patterns of change. Many patients have unrealistic expectations about the duration of therapy; they expect a quick recovery in a small number of therapy (see table 3). There is extensive evidence that the expected number of sessions is considered the best predictor of the actual number of sessions attended (e.g. Mueller \& Pekarik, 2000) and that this expectation also influences the dropout rate (e.g. Callahan et al., 2009). In their RCT with 63 participants, Swift and Callahan (2011) found that the implementation of an education program that informs patients about the anticipated duration of therapy and the positive relationship between the number of therapy sessions and the probability of recovery prolongs the absolute duration of treatment and reduces the dropout rate. Therefore, Swift and Callahan provide first evidence of an effective strategy to reduce dropout rates.

Secondly, the patient's role expectations must be clarified. Existing expectations as well as possible misconceptions must be addressed. Swift et al. (2012) assume that unmet role expectations can either result from naïve beliefs shaped, for example, by stereotypes, but they also occur when the patient has a precise idea that cannot be fulfilled by his therapist. There is extensive evidence that these unmet role expectations can predict dropout (e.g. Callahan et al., 2009). To prevent possible premature discontinuation of therapy, it is therefore important to discuss the distribution of roles in the first therapy session. There are numerous studies showing the effectiveness of different role induction strategies in psychological treatments, especially of so-called orientation videos (Swift et al., 2012). For example, Reis and Brown (2006) showed in their interventions study with 125 patients that dropout could be significantly reduced by viewing a 12-minute role induction video. While the video format is often used to introduce the different roles, there is also evidence from older studies showing that this can also be done orally or in 
written format (Swift et al., 2012). These findings are strengthened by Barrett et al.'s (2008) systematic literature review. The authors emphasize the importance of an appropriate preparation of the patient for the therapy and cite various study findings to prove their point. In conclusion, there is extensive evidence from several systematic reviews as well as numerous intervention studies indicating the effectiveness of role induction strategies (e.g. Ogrodniczuk et al., 2005)

Third, based on their meta-analytic findings on the occurrence of dropout Swift et al. (2012) suggest that it is important to incorporate patients' preferences before starting treatment including the type of treatment, but also preferences regarding to the therapist, therapeutic tools, behavior, and roles. According to the authors, patients often have concrete ideas, without having full insight into the possible treatment options. Therefore, therapists should share their knowledge of different therapeutic options with the patient, listen to the patient's preferences and make a joint decision on the best approach (Swift et al., 2012). Meta-analytic findings including 18 studies show that patients whose preferences were addressed, discontinued therapy less frequently than patients who had no choice or were provided with a treatment option they did not prefer (Swift et al., 2011) (see Table 3). To our knowledge there are no studies investigating the effectiveness of strategies to incorporate patients' preference.

Fourth, a handful of studies have shown that building and strengthening patients' hope is a central factor for therapeutic success and thus, it is assumed to be effective in the reduction of dropout (Swift et al., 2012). Swift et al. (2012) recommend that it is important to ensure that a conviction that the therapy will contribute to the improvement of the problems is given, but at the same time, no unrealistic expectations are reinforced. Constantino et al. (2011) describe two strategies to build such a realistic hope. One is that the therapist describes a treatment rational that is comprehensible for the patient and thereby draws on striking research results if necessary. On the other hand, the therapist expresses his personal confidence in the effectiveness of the therapy and in the patient's abilities. Again, there is no evidence actual testing strategies focusing explicitly on patients' hope. Most of the authors rather focus on giving suggestions on this topic.

Fifth, it is essential to foster the therapeutic alliance (see Table 3). Working with an understanding therapist where there is agreement on goals and tasks and where a strong sense of cohesion dominates, enhances the perceived benefits of therapy. Considering the extensive evidence on the influence of therapeutic alliance on treatment outcome, Swift et al. (2012) suggest that such a strong therapist-patient relationship reduces the probability of premature discontinuation of therapy. In their meta-analysis with 11 included studies, Sharf et al. (2010) were able to prove this connection between high dropout rates and low therapeutic alliance. Based on these finding Swift et al. (2012) point out to the importance of establishing strategies to strengthen the alliance for an effective reduction of dropout. According to the authors fostering the therapeutic alliance not only refers to the first therapeutic session but is equally important in the course of therapy. They describe different strategies that are being used in the two phases to strengthen and later maintain the therapeutic alliance. While at the beginning of the therapy, an 
agreement on goals and tasks should be achieved throughout therapy strategies for the development of a strong bond become increasingly important. Therapists should then take care to create a safe and empathic environment in which patients feel that they are working together on the same goal (Swift et al., 2012). There is a consensus in the current literature that it is particularly important to be aware of the fact that a break in the therapeutic relationship is often experienced as a severe loss, resulting in a higher risk for dropout (e.g. Barrett et al., 2008). Even though it is sometimes difficult to detect them, such ruptures should always be taken into account. Several authors suggest that attention must be paid to the patient's negative feelings in order to work together to resolve the conflict and re-strengthen the relationship (Ogrodniczuk et al., 2005; Safran et al., 2011). Research investigating the effects of applied specific strategies to foster the alliance on the dropout rate is missing so far.

Sixth, there is extensive evidence supporting that assessing and discussing the treatment progress is indispensable to allow a patient-focused approach in the course of treatment (Swift et al., 2012). To realize a strategic monitoring, objective measures to track the outcome are used, the patient's progress in treatment is compared with typical patterns of improvement, and finally the therapist is given feedback on the relation of the present therapy progress to the desired progress (Howard et al., 1996). Michael J. Lambert et al. (2001) state that this monitoring is important because a deviating therapy progress is often accompanied by negative feelings of the patient and thus dropout becomes more likely. Swift et al. (2012) argue that as a result, the patient no longer perceives the benefits of the therapy; he feels that the therapy is stagnating and that the costs incurred outweigh the benefits. If the therapist detects these changes at an early stage, adjustments can be made to the course of therapy before premature discontinuation can even occur. In addition to the use of an objective monitoring system, the authors suggest that it may also proves to be effective to directly talk to the patient about the topic and ask how he perceives the course of therapy. Although Michael J. Lambert and Shimokawa (2011) review the use of feedback systems on treatment outcome, to our knowledge there exists no research on the effects on patients' dropout.

In their review of literature, Barrett et al. (2008) discuss another strategy that can help to reduce dropout complementing the recommendations given by Swift et al. (2012). As motivation can be seen as crucial for beginning and remaining in a treatment, this factor is also important when talking of premature termination of therapy (Barrett et al., 2008). The authors suggest that this results in the need for a treatment approach aimed at increasing and facilitating the internal motivation of patients to achieve changes. Barrett et al. (2008) focus on strategies like motivational interviewing. By helping the patient to build up their own motivation of change, they are guided to resolve possible ambivalent feelings towards the therapy. An internally motivated change process can therefore increase commitment and reduce dropout rates, so the authors. There is first evidence that motivational interviewing proves to be effective in reducing dropout 
rates (Carroll et al., 2001). However it should be noted that despite the positive effects, results on motivational interviewing are inconsistent (Barrett et al., 2008).

Furthermore, it appears to be important to look at patient characteristics correlated with high dropout rates to derive adequate strategies to reduce dropout. Research on predictors of dropout has shown that dropout might occurs more frequently among younger, less educated patients, who may have a higher risk of being in dire of financial straits. Barrett et al. (2008) suggest that sometimes these individuals are facing urgent crises that bring them to seek treatment. Once this crisis is solved, other upcoming needs might outweigh the need to attend treatment and dropout becomes more likely. Some authors hypothesize that, to prevent dropout, strategies are needed that focus on the development of a skill set, helping these patients to maintain in the treatment (e.g. Ogrodniczuk et al., 2005). According to numerous studies the offer of a so-called preparatory intervention are effective in reducing dropout (see Ogrodniczuk et al., 2005, for an overview). Though these interventions include various aspects of preparation, including role induction as described earlier. As no specific mechanisms of action can be retrieved, a link to reinforcement of problematic patient characteristics is more hypothetical.

Derived from the clinical practice, sending appointment reminders might be another strategy that can help in reducing premature termination of dropout. Ogrodniczuk et al. (2005) suggest that by occasionally sending quick reminders, the patients' responsibility in attending a scheduled session is addressed and therefore dropout may become less likely. Although there is no wellfunded research on the use of appointment reminders, this strategy is a low-cost mean that can be implemented easily. Further, this strategy might be especially effective when treating refugees and asylum seekers that are not used to the western health care system.

\subsection{Specific strategies for refugees and asylum seekers}

In the treatment of refugees and asylum seekers, it is further important to implement specific strategies adapted to the particular treatment challenges and needs of this people group (see Table 3, for an overview).

As described earlier, there are significant cultural differences in the perception of mental illness, psychological treatment, and therapists as well as treatment expectations that appear to contribute to dropout in refugee samples. Addressing these disparities therefore appears promising to reduce dropout in this group. A keyword in this context is the development and promotion of cultural competencies (Barrett et al., 2008; Liedl et al., 2016; Maramba \& Nagayama Hall, 2002). Some authors suggest that building cultural competencies in treatment providers includes raising awareness and generating knowledge about cultural differences and further acquiring skills to deal with them. Therapists acquire suitable strategies for role inductions and learn about dealing with deviating expectations and goals. The acquisition of cultural skills thus leads to a responsive approach to the needs and expectations of the patients and strengthens the therapeutic alliance (Barrett et al., 2008; Liedl et al., 2016). Although this might consequently 
reduce the risk of premature discontinuation of therapy, studies investigating the effectiveness of cultural competencies on dropout are still missing (van Loon et al., 2011).

Further, Liedl et al. (2016) recommend a detailed assessment of the patients history (anamnesis), extended by culture- and refugee-specific aspects. This measure allows a consideration of additional, refugee-specific areas and enables the exploration of culture-specific beliefs about mental illnesses and psychotherapeutic treatment. An early clarification of barriers that could complicate the therapeutic process allows an adaption of the process before premature termination can even occur (Liedl et al., 2016). In the further course of the therapy, a culturally sensitive approach should be chosen. This takes the specific symptoms, their history and individual biography into account, breaks down cultural misunderstandings, and attempts to overcome language barriers (Abdallah-Steinkopff \& Soyer, 2013; Kahraman \& AbdallahSteinkopff, 2010). However, the effects of a detailed anamnesis and a culturally sensitive approach on dropout rates have not been studied yet.

The difficult living conditions and the presence of multiple post-migration stressors must also be considered, as these factors might influence the probability of premature discontinuation of therapy (Liedl et al., 2016). Accordingly, strategies addressing the mentioned obstacles must be developed for comprehensive prevention of dropout. Ogrodniczuk et al. (2005) discuss the use of case management. In addition to the actual psychological treatment, so-called case managers can help the patient to cope with the daily stressful living conditions, the presence of which might otherwise have led to a premature termination of therapy. One study reported that case management in addition to CBT reduced the dropout rate by $50 \%$ compared to CBT alone (Miranda et al., 2003). Furthermore, the therapeutic process should also focus on dealing with the difficult daily life and the existing post-migration stressors. Liedl et al. (2016) suggest that a resource-oriented approach as well as the increase of pleasant activities and the encouragement of social integration is indispensable, especially at the beginning of the therapy. Such a consideration of the living conditions can not only contribute to a stabilization of the patients, but can also create a feeling of being understood, which in turn strengthens the compliance (Liedl et al., 2016; Priebe et al., 2016).

Although a detailed consideration of the challenges of working with interpreters would go beyond the scope of this guideline, it is important to note that working with interpreters can lead to difficulties, which may increase the risk of premature termination of therapy (Abdallah-Steinkopff, 2020; Liedl et al., 2016). Conflicting political, ethical or religious affiliations, or in some cases a mismatch with regard to gender or the handling of taboo subjects, are seen as problematic (Liedl et al., 2016). It is important to address critical points at an early stage, but also when they occur later in the course of therapy, in order to prevent possible ruptures in the therapeutic relationship. Special attention should be drawn to the interpreters' duty of confidentiality (Abdallah-Steinkopff, 2020; Liedl et al., 2016). Since the exile community is mostly rather small, there is otherwise the danger that the patient will develop inhibitions to address critical issues. 
The effects of a mismatch between patients and interpreters are derived from clinical practices, again well-founded research is missing.

Table 3: Key findings on preventing dropout

\begin{tabular}{l|l}
\hline \multicolumn{2}{l}{ General strategies } \\
\hline & Inform patient about duration of treatment and typical patterns of change \\
\hline & Clarify role expectations and misconceptions \\
\hline & Incorporate the preferences of the patient \\
\hline & Build and strengthen hope \\
\hline & Foster the therapeutic alliance \\
\hline & Assess and discuss the treatment progress \\
\hline & Increase and facilitate the internal motivation \\
\hline \multirow{2}{*}{ Specific strategies } \\
\hline & $\begin{array}{l}\text { Develop and promote cultural competencies: raising awareness, generating } \\
\text { knowledge, and acquiring skills }\end{array}$ \\
\hline & \begin{tabular}{l} 
Think about the additional use of case management \\
\hline
\end{tabular} \\
\hline & $\begin{array}{l}\text { Focus on a resource-oriented approach, increase pleasant activities, and } \\
\text { encourage social integration }\end{array}$ \\
\hline & Keep in mind the challenges of working with interpreters \\
\hline
\end{tabular}

\section{Conclusion}

In this guideline, we have described the multiple psycho-social stressors, the experienced insecurity and resulting mental health burden refugees and asylum seekers resettled in highincome countries are facing. Further, we have looked at the current evidence on the effectiveness of psychological and psychosocial treatments for this people group. It is important to note that empirical research on dropout in general as well as specifically in the treatment of refugees and asylum seekers is limited. While prevalence rates of about $20 \%$ (with a substantial variability) are reported for dropout from psychological treatment, there is currently no reliable data on dropout in treatment of refugees. However, there are reasons to assume that dropout rates are even higher in this group. It therefore appears highly important to develop and empirically test measures to reduce dropout in treatment, with research into predictors and subjective reasons for dropout as an important first step.

Predictive factors for dropout include sociodemographic variables as well as variables concerning patient's diagnosis, treatment characteristics, therapist variables, and study and design variables. 
Subjective reasons for dropout include insufficient motivation for therapy or motivation of change, dissatisfaction with the therapist, therapy or change of symptoms and external barriers.

In the current literature, preventive measures are mostly derived from research on predictors or refer to recommendations from the clinical practice. There are hardly any empirical studies testing the effects of these measures on the dropout rate. However, there is reliable evidence for a reduction of dropout through an appropriate preparation of the patient for psychological treatment. This preparation mainly focuses on role induction, but it also includes other aspects that can have a positive effect on the dropout rate. These are for example information on psychological treatments in general (duration, change processes) and a strengthening of the therapeutic alliance. Adequate preparation can also increase therapy motivation and hope and further contribute to the development of a skill set. Due to the complex interaction of the individual aspects, however, no causal relation can be derived. Conclusions on other factors therefore remain hypothetical. Future research should focus on a differentiated investigation of specified strategies to reduce dropout.

In the treatment of refugees and asylum seekers strategies to reduce dropout are also mainly based on recommendations based on clinical consensus. Especially the development and promotion of cultural competencies and the adaptation of treatment to the specific needs of the patients (post-migration stressors, difficult living conditions) have been proposed to have a positive effect on the dropout rate.

The detailed presentation of the current evidence regarding prevalence, predictors and established preventive measures is intended to draw attention to the importance of the dropout phenomenon. In addition, it serves as a guideline for clinical researchers and therapists, and points out to the need of further research. A meta-analysis that is currently in preparation by the authors of this guideline can be seen as a first step towards research on dropout in the treatment of refugees and asylum seekers. 


\section{References}

Abdallah-Steinkopff, B. (2020, February 20-22). Prävention von Dropout bei Maßnahmen mit Geflüchteten [Workshop Presentation]. Workshop: Prävention von Dropout bei Maßnahmen mit Geflüchteten, (Munich).

Abdallah-Steinkopff, B., \& Soyer, J. (2013). Traumatisierte Flüchtlinge. Kultursensible Psychotherapie im politischen Spannungsfeld. In J. Feldmann Robert E. \& G. H. Seidler (Eds.), Traum(a) Migration: Aktuelle Konzepte zur Therapie traumatisierter Flüchtlinge und Folteropfer (1st ed., pp. 137-166). Psychosozial-Verlag. https://doi.org/10.30820/9783837966558-137

Alpak, G., Unal, A., Bulbul, F., Sagaltici, E., Bez, Y., Altindag, A., Dalkilic, A., \& Savas, H. A. (2015). Post-traumatic stress disorder among Syrian refugees in Turkey: a cross-sectional study. International Journal of Psychiatry in Clinical Practice, 19(1), 45-50. https://doi.org/10.3109/13651501.2014.961930

Baekeland, F., \& Lundwall, L. (1975). Dropping out of treatment: A critical review. In Psychological Bulletin (Vol. 82, Issue 5, pp. 738-783). American Psychological Association. https://doi.org/10.1037/h0077132

Barrett, M. S., Chua, W.-J., Crits-Christoph, P., Gibbons, M. B., Casiano, D., \& Thompson, D. (2008). Early withdrawl from mental health treatment: Implications for psychotherapy practice. Psychotherapy (Chicago, III.), 45(2), 247-267. https://doi.org/10.1037/00333204.45.2.247

Beckham, E. E. (1992). Predicting patient dropout in psychotherapy. In Psychotherapy: Theory, Research, Practice, Training (Vol. 29, Issue 2, pp. 177-182). Division of Psychotherapy (29), American Psychological Association. https://doi.org/10.1037/0033-3204.29.2.177

Bjork, T., Bjorck, C., Clinton, D., Sohlberg, S., \& Norring, C. (2009). What happened to the ones who dropped out? Outcome in eating disorder patients who complete or prematurely terminate treatment. European Eating Disorders Review: The Journal of the Eating Disorders Association, 17(2), 109-119. https://doi.org/10.1002/erv.911

Bogic, M., Ajdukovic, D., Bremner, S., Franciskovic, T., Galeazzi, G. M., Kucukalic, A., LecicTosevski, D., Morina, N., Popovski, M., Schutzwohl, M., Wang, D., \& Priebe, S. (2012). Factors associated with mental disorders in long-settled war refugees: refugees from the former Yugoslavia in Germany, Italy and the UK. The British Journal of Psychiatry: The Journal of Mental Science, 200(3), 216-223. https://doi.org/10.1192/bjp.bp.110.084764

Böttche, M., Heeke, C., \& Knaevelsrud, C. (2016). Sequenzielle Traumatisierungen, Traumafolgestörungen und psychotherapeutische Behandlungsansätze bei kriegstraumatisierten erwachsenen Flüchtlingen in Deutschland. BundesgesundheitsblattGesundheitsforschung-Gesundheitsschutz, 59(5), 621-626. 
Boyd-Franklin, N. (2013). Black families in therapy: Understanding the African American experience. Guilford Publications.

Cahill, J., Barkham, M., Hardy, G., Rees, A., Shapiro, D. A., Stiles, W. B., \& Macaskill, N. (2003). Outcomes of patients completing and not completing cognitive therapy for depression. British Journal of Clinical Psychology, 42(2), 133-143. https://doi.org/10.1348/014466503321903553

Callahan, J. L., Aubuchon-Endsley, N., Borja, S. E., \& Swift, J. K. (2009). Pretreatment expectancies and premature termination in a training clinic environment. Training and Education in Professional Psychology, 3(2), 111.

Carroll, K. M., Libby, B., Sheehan, J., \& Hyland, N. (2001). Motivational interviewing to enhance treatment initiation in substance abusers: an effectiveness study. The American Journal on Addictions, 10(4), 335-339. https://doi.org/10.1080/aja.10.4.335.339

Cinkaya, F. (2016). Die Vielfalt von Abbrüchen in der Psychotherapie. Eine Metaanalyse. The Multiplicity of Dropouts in Psychotherapy: A Meta-Analysis. Kovac.

Constantino, M. J., Glass, C. R., Arnkoff, D. B., Ametrano, R. M., \& Smith, J. Z. (2011). Expectations. In Psychotherapy relationships that work: Evidence-based responsiveness, 2nd ed. (pp. 354-376). Oxford University Press. https://doi.org/10.1093/acprof:oso/9780199737208.003.0018

Crumlish, N., \& O'Rourke, K. (2010). A systematic review of treatments for post-traumatic stress disorder among refugees and asylum-seekers. The Journal of Nervous and Mental Disease, 198(4), 237-251. https://doi.org/10.1097/NMD.0b013e3181d61258

Ebner, G. (2001). Grundlagen transkultureller Begutachtung. In T. Hegemann \& R. Salman (Eds.), Transkulturelle Psychiatrie (pp. 232-251). Köln: Psychiatrie Verlag.

Farber, B. A. (1983). Psychotherapists' perceptions of stressful patient behavior. Professional Psychology: Research and Practice, 14(5), 697-705. https://doi.org/10.1037/07357028.14.5.697

Fazel, M., Wheeler, J., \& Danesh, J. (2005). Prevalence of serious mental disorder in 7000 refugees resettled in western countries: a systematic review. Lancet (London, England), 365(9467), 1309-1314. https://doi.org/10.1016/S0140-6736(05)61027-6

Fernandez, E., Salem, D., Swift, J. K., \& Ramtahal, N. (2015). Meta-analysis of dropout from cognitive behavioral therapy: Magnitude, timing, and moderators. Journal of Consulting and Clinical Psychology, 83(6), 1108-1122. https://doi.org/10.1037/ccp0000044

Gammouh, O. S., Al-Smadi, A. M., Tawalbeh, L. I., \& Khoury, L. S. (2015). Chronic diseases, lack of medications, and depression among Syrian refugees in Jordan, 2013-2014. Preventing Chronic Disease, 12, E10. https://doi.org/10.5888/pcd12.140424 
Garfield, S. L. (1986). Research on client variables in psychotherapy. Handbook of Psychotherapy and Behavior Change, 6, 213-256.

Garfield, S. L. (1994). Research on client variables in psychotherapy. In Handbook of psychotherapy and behavior change, 4th ed. (pp. 190-228). John Wiley \& Sons.

Gunderson, J. G., Frank, A. F., Ronningstam, E. F., Wachter, S., Lynch, V. J., \& Wolf, P. J. (1989). Early discontinuance of borderline patients from psychotherapy. Journal of Nervous and Mental Disease.

Hargreaves, S. (2002). A body of evidence: torture among asylum seekers to the West. Lancet (London, England), 359(9308), 793-794. https://doi.org/10.1016/S0140-6736(02)07888-1

Hatchett, G. T., Han, K., \& Cooker, P. G. (2002). Predicting premature termination from counseling using the Butcher Treatment Planning Inventory. In Assessment (Vol. 9, Issue 2, pp. 156-163). Sage Publications. https://doi.org/10.1177/10791102009002006

Hatchett, G. T., \& Park, H. L. (2003). Comparison of Four Operational Definitions of Premature Termination. In Psychotherapy: Theory, Research, Practice, Training (Vol. 40, Issue 3, pp. 226-231). Educational Publishing Foundation. https://doi.org/10.1037/0033-3204.40.3.226

Hollifield, M., Warner, T. D., Lian, N., Krakow, B., Jenkins, J. H., Kesler, J., Stevenson, J., \& Westermeyer, J. (2002). Measuring trauma and health status in refugees: a critical review. JAMA, 288(5), 611-621. https://doi.org/10.1001/jama.288.5.611

Horner, M. S., \& Diamond, D. (1996). Object relations development and psychotherapy dropout in borderline outpatients. In Psychoanalytic Psychology (Vol. 13, Issue 2, pp. 205-223). Lawrence Erlbaum Associates, Inc. https://doi.org/10.1037/h0079648

Howard, K. I., Moras, K., Brill, P. L., Martinovich, Z., \& Lutz, W. (1996). Evaluation of psychotherapy: Efficacy, effectiveness, and patient progress. In American Psychologist (Vol. 51, Issue 10, pp. 1059-1064). American Psychological Association. https://doi.org/10.1037/0003-066X.51.10.1059

Imel, Z. E., Laska, K., Jakupcak, M., \& Simpson, T. L. (2013). Meta-Analysis of Dropout in Treatments for Posttraumatic Stress Disorder. Journal of Consulting and Clinical Psychology, 81(3), 394-404. https://doi.org/10.1037/a0031474

Kahraman, B., \& Abdallah-Steinkopff, B. (2010). Same same but different. PiD - Psychotherapie im Dialog, 11(04), 306-312. https://doi.org/10.1055/s-0030-1265905

Kalt, A., Hossain, M., Kiss, L., \& Zimmerman, C. (2013). Asylum seekers, violence and health: a systematic review of research in high-income host countries. American Journal of Public Health, 103(3), e30-42. https://doi.org/10.2105/AJPH.2012.301136

Kip, A., Priebe, S., Holling, H., \& Morina, N. (2020). Psychological interventions for posttraumatic stress disorder and depression in refugees: A meta-analysis of randomized controlled trials. Clinical Psychology \& Psychotherapy. https://doi.org/10.1002/cpp.2446 
Knox, S., Adrians, N., Everson, E., Hess, S., Hill, C., \& Crook-Lyon, R. (2011). Clients' perspectives on therapy termination. Psychotherapy Research: Journal of the Society for Psychotherapy Research, 21(2), 154-167. https://doi.org/10.1080/10503307.2010.534509

Kolb, D. L., Beutler, L. E., Davis, C. S., Crago, M., \& Shanfield, S. B. (1985). Patient and therapy process variables relating to dropout and change in psychotherapy. In Psychotherapy: Theory, Research, Practice, Training (Vol. 22, Issue 4, pp. 702-710). Division of Psychotherapy (29), American Psychological Association. https://doi.org/10.1037/h0085556

Lambert, J. E., \& Alhassoon, O. M. (2015). Trauma-focused therapy for refugees: meta-analytic findings. Journal of Counseling Psychology, 62(1), 28-37. https://doi.org/10.1037/cou0000048

Lambert, M. J. (2007). Presidential address: What we have learned from a decade of research aimed at improving psychotherapy outcome in routine care. Psychotherapy Research, 17(1), $1-14$.

Lambert, M. J., Hansen, N. B., \& Finch, A. E. (2001). Patient-focused research: Using patient outcome data to enhance treatment effects. In Journal of Consulting and Clinical Psychology (Vol. 69, Issue 2, pp. 159-172). American Psychological Association. https://doi.org/10.1037/0022-006X.69.2.159

Lambert, M. J., \& Shimokawa, K. (2011). Collecting client feedback. In Psychotherapy relationships that work: Evidence-based responsiveness, 2nd ed. (pp. 203-223). Oxford University Press. https://doi.org/10.1093/acprof:oso/9780199737208.003.0010

Liedl, A., Böttche, M., Abdallah-Steinkopff, B., \& Knaevelsrud, C. (2016). Psychotherapie mit Flüchtlingen-neue Herausforderungen, spezifische Bedürfnisse: Das Praxisbuch für Psychotherapeuten und Ärzte. Schattauer Verlag.

Maher, M. J., Huppert, J. D., Chen, H., Duan, N., Foa, E. B., Liebowitz, M. R., \& Simpson, H. B. (2010). Moderators and predictors of response to cognitive-behavioral therapy augmentation of pharmacotherapy in obsessive-compulsive disorder. Psychological Medicine, 40(12), 2013-2023. https://doi.org/10.1017/S0033291710000620

Maramba, G. G., \& Nagayama Hall, G. C. (2002). Meta-analyses of ethnic match as a predictor of dropout, utilization, and level of functioning. In Cultural Diversity and Ethnic Minority Psychology (Vol. 8, Issue 3, pp. 290-297). Educational Publishing Foundation. https://doi.org/10.1037/1099-9809.8.3.290

McMurran, M., Huband, N., \& Overton, E. (2010). Non-completion of personality disorder treatments: A systematic review of correlates, consequences, and interventions. Clinical Psychology Review, 30, 277-287. https://doi.org/10.1016/j.cpr.2009.12.002

Miranda, J., Azocar, F., Organista, K. C., Dwyer, E., \& Areane, P. (2003). Treatment of Depression Among Impoverished Primary Care Patients From Ethnic Minority Groups. Psychiatric 
Services, 54(2), 219-225. https://doi.org/10.1176/appi.ps.54.2.219

Mueller, M., \& Pekarik, G. (2000). Treatment duration prediction: Client accuracy and its relationship to dropout, outcome, and satisfaction. In Psychotherapy: Theory, Research, Practice, Training (Vol. 37, Issue 2, pp. 117-123). Division of Psychotherapy (29), American Psychological Association. https://doi.org/10.1037/h0087701

Nickerson, A., Bryant, R. A., Silove, D., \& Steel, Z. (2011). A critical review of psychological treatments of posttraumatic stress disorder in refugees. Clinical Psychology Review, 31(3), 399-417. https://doi.org/10.1016/j.cpr.2010.10.004

Nose, M., Ballette, F., Bighelli, I., Turrini, G., Purgato, M., Tol, W., Priebe, S., \& Barbui, C. (2017). Psychosocial interventions for post-traumatic stress disorder in refugees and asylum seekers resettled in high-income countries: Systematic review and meta-analysis. PLOS ONE, 12(2). https://doi.org/10.1371/journal.pone.0171030

Ogrodniczuk, J. S., Joyce, A. S., \& Piper, W. E. (2005). Strategies for Reducing Patient-Initiated Premature Termination of Psychotherapy. Harvard Review of Psychiatry, 13(2), 57-70. https://doi.org/10.1080/10673220590956429

Palic, S., \& Elklit, A. (2011). Psychosocial treatment of posttraumatic stress disorder in adult refugees: A systematic review of prospective treatment outcome studies and a critique. Journal of Affective Disorders, 131, 8-23. https://doi.org/10.1016/j.jad.2010.07.005

Parsonage, M. (2003). The economic and social costs of mental illness.

Pekarik, G. (1992). Relationship of clients' reasons for dropping out of treatment to outcome and satisfaction. Journal of Clinical Psychology, 48(1), 91-98.

Piselli, A., Halgin, R., \& MacEwan, G. (2011). What went wrong? Therapists' reflections on their role in premature termination. Psychotherapy Research, 21(4), 400-415. http://10.0.4.56/10503307.2011.573819

Porter, M., \& Haslam, N. (2005). Predisplacement and postdisplacement factors associated with mental health of refugees and internally displaced persons: a meta-analysis. JAMA, 294(5), 602-612. https://doi.org/10.1001/jama.294.5.602

Priebe, S., Giacco, D., \& El-Nagib, R. (2016). Public health aspects of mental health among migrants and refugees: a review of the evidence on mental health care for refugees, asylum seekers and irregular migrants in the WHO European Region. World Health Organization. Regional Office for Europe.

Reis, B., \& Brown, L. (2006). Preventing Therapy Dropout in the Real World: The Clinical Utility of Videotape Preparation and Client Estimate of Treatment Duration. Professional Psychology: Research and Practice, 37, 311-316. https://doi.org/10.1037/07357028.37.3.311 
Roos, J., \& Werbart, A. (2013). Therapist and relationship factors influencing dropout from individual psychotherapy: a literature review. Psychotherapy Research : Journal of the Society for Psychotherapy Research, 23(4), 394-418. https://doi.org/10.1080/10503307.2013.775528

Ryan, D., Dooley, B., \& Benson, C. (2008). Theoretical Perspectives on Post-Migration Adaptation and Psychological Well-Being among Refugees: Towards a Resource-Based Model. Journal of Refugee Studies, 21. https://doi.org/10.1093/jrs/fem047

Safran, J. D., Muran, J. C., \& Eubanks-Carter, C. (2011). Repairing alliance ruptures. In Psychotherapy relationships that work: Evidence-based responsiveness, 2nd ed. (pp. 224238). Oxford University Press. https://doi.org/10.1093/acprof:oso/9780199737208.003.0011

Sandhu, S., Bjerre, N. V, Dauvrin, M., Dias, S., Gaddini, A., Greacen, T., Ioannidis, E., Kluge, U., Jensen, N. K., Lamkaddem, M., Puigpinós i Riera, R., Kósa, Z., Wihlman, U., Stankunas, M., Straßmayr, C., Wahlbeck, K., Welbel, M., \& Priebe, S. (2013). Experiences with treating immigrants: a qualitative study in mental health services across 16 European countries. Social Psychiatry and Psychiatric Epidemiology, 48(1), 105-116. https://doi.org/10.1007/s00127-012-0528-3

Sharf, J., Primavera, L. H., \& Diener, M. J. (2010). Dropout and therapeutic alliance: A metaanalysis of adult individual psychotherapy. In Psychotherapy: Theory, Research, Practice, Training (Vol. 47, Issue 4, pp. 637-645). Educational Publishing Foundation. https://doi.org/10.1037/a0021175

Slewa-Younan, S., Guajardo, M. G. U., Heriseanu, A., \& Hasan, T. (2015). A systematic review of post-traumatic stress disorder and depression amongst Iraqi refugees located in western countries. Journal of Immigrant and Minority Health, 17(4), 1231-1239.

Slobodin, O., \& de Jong, J. T. V. M. (2015). Mental health interventions for traumatized asylum seekers and refugees: What do we know about their efficacy? International Journal of Social Psychiatry, 61(1), 17-26. https://doi.org/10.1177/0020764014535752

Steel, Z., Chey, T., Silove, D., Marnane, C., Bryant, R. A., \& van Ommeren, M. (2009). Association of torture and other potentially traumatic events with mental health outcomes among populations exposed to mass conflict and displacement: a systematic review and metaanalysis. JAMA, 302(5), 537-549. https://doi.org/10.1001/jama.2009.1132

Swift, J. K., \& Callahan, J. L. (2011). Decreasing treatment dropout by addressing expectations for treatment length. Psychotherapy Research, 21(2), 193-200. https://doi.org/10.1080/10503307.2010.541294

Swift, J. K., Callahan, J. L., \& Vollmer, B. M. (2011). Preferences. In Psychotherapy relationships that work: Evidence-based responsiveness, 2nd ed. (pp. 301-315). Oxford University Press. https://doi.org/10.1093/acprof:oso/9780199737208.003.0015 
Swift, J. K., Callahan, J., \& Levine, J. C. (2009). Using clinically significant change to identify premature termination. Psychotherapy (Chicago, III.), 46(3), 328-335. https://doi.org/10.1037/a0017003

Swift, J. K., \& Greenberg, R. P. (2012). Premature discontinuation in adult psychotherapy: a metaanalysis. Journal of Consulting and Clinical Psychology, 80(4), 547-559. https://doi.org/10.1037/a0028226

Swift, J. K., \& Greenberg, R. P. (2014). A treatment by disorder meta-analysis of dropout from psychotherapy. Journal of Psychotherapy Integration, 24(3), 193.

Swift, J. K., Greenberg, R. P., Tompkins, K. A., \& Parkin, S. R. (2017). Treatment refusal and premature termination in psychotherapy, pharmacotherapy, and their combination: A metaanalysis of head-to-head comparisons. Psychotherapy (Chicago, III.), 54(1), 47-57. https://doi.org/10.1037/pst0000104

Swift, J. K., Greenberg, R. P., Whipple, J., \& Kominiak, N. (2012). Practice Recommendations for Reducing Premature Termination in Therapy. Professional Psychology: Research and Practice, 43, 379-387. https://doi.org/10.1037/a0028291

Thompson, C. T., Vidgen, A., \& Roberts, N. P. (2018). Psychological interventions for posttraumatic stress disorder in refugees and asylum seekers: A systematic review and metaanalysis. Clinical Psychology Review, 63, 66-79. https://doi.org/10.1016/j.cpr.2018.06.006

Tribe, R. H., Sendt, K.-V., \& Tracy, D. K. (2019). A systematic review of psychosocial interventions for adult refugees and asylum seekers. Journal of Mental Health, 28(6), 662-676. https://doi.org/10.1080/09638237.2017.1322182

Turrini, G., Purgato, M., Acarturk, C., Anttila, M., Au, T., Ballette, F., Bird, M., Carswell, K., Churchill, R., Cuijpers, P., Hall, J., Hansen, L. J., Kösters, M., Lantta, T., Nosè, M., Ostuzzi, G., Sijbrandij, M., Tedeschi, F., Valimaki, M., ... Barbui, C. (2019). Efficacy and acceptability of psychosocial interventions in asylum seekers and refugees: Systematic review and metaanalysis. Epidemiology and Psychiatric Sciences, 28(4), 376-388.

https://doi.org/10.1017/S2045796019000027

Turrini, G., Purgato, M., Ballette, F., Nose, M., Ostuzzi, G., \& Barbui, C. (2017). Common mental disorders in asylum seekers and refugees: umbrella review of prevalence and intervention studies. International Journal of Mental Health Systems, 11, 51.

https://doi.org/10.1186/s13033-017-0156-0

UNHCR. (2019). Global Trends. Forced displacement in 2018.

van Loon, A., van Schaik, D. J. F., Dekker, J. J., \& Beekman, A. T. F. (2011). Effectiveness of an intercultural module added to the treatment guidelines for Moroccan and Turkish patients with depressive and anxiety disorders. BMC Psychiatry, 11, 13.

https://doi.org/10.1186/1471-244X-11-13 
Westmacott, R., Hunsley, J., Best, M., Rumstein-McKean, O., \& Schindler, D. (2010). Client and therapist views of contextual factors related to termination from psychotherapy: $A$ comparison between unilateral and mutual terminators. Psychotherapy Research, 20(4), 423-435. http://10.0.4.56/10503301003645796

Wierzbicki, M., \& Pekarik, G. (1993). A meta-analysis of psychotherapy dropout. In Professional Psychology: Research and Practice (Vol. 24, Issue 2, pp. 190-195). American Psychological Association. https://doi.org/10.1037/0735-7028.24.2.190

Winkler, R. (2018). Dropout in Psychotherapien. LBI-HTA Projektbericht Nr.:100.

Zimmermann, D., Rubel, J., Page, A. C., \& Lutz, W. (2017). Therapist effects on and predictors of non-consensual dropout in psychotherapy. Clinical Psychology \& Psychotherapy, 24(2), 312321. 


\section{List of tables}

Table 1: Definition and operationalization of dropout ................................. 7

Table 2: Key findings on predictors and subjective reasons for dropout ............... 15

Table 3: Key findings on preventing dropout ....................................... 21 